\title{
Elecciones 2015 y 2016 en España: el debate desde los temas a los "meta-temas" de agenda
}

\author{
Elections in Spain in 2015 and 2016: \\ the agenda debate from topics to "meta-topics"
}

Raquel Rodríguez-Díaz. Universidad Rey Juan Carlos. España. raquel.rodriguez@urjc.es

$[\underline{\mathrm{CV}}]$ (1) G

Antón R. Castromil. Universidad Complutense. España.

arcastromil@,ccinf.ucm.es

$[\underline{\mathrm{CV}}]$ (1) $\mathrm{G}$

Este trabajo forma parte del proyecto de investigación "DEBATv, Debates electorales televisados en España: modelos, proceso, diagnóstico y propuesta" (CSO2017-83159-R), proyecto de $I+D+i$ (Retos) financiado por el Ministerio de Ciencia, Innovación y Universidades y por la Agencia Estatal de Investigación (AEI) del Gobierno de España, con el apoyo del Fondo Europeo de Desarrollo Regional (Feder) de la Unión Europea (UE).

Queremos expresar nuestro agradecimiento a Ana Jiménez Pérez su colaboración en el análisis de contenido.

Cómo citar este artículo / Referencia normalizada

Rodríguez-Díaz, R. y Castromil, A. R. (2020). Elecciones 2015 y 2016 en España: el debate desde los temas a los "meta-temas" de agenda. Revista Latina de Comunicación Social, (76), 209-227. https://www.doi.org/10.4185/RLCS-2020-1444

\section{RESUMEN}

Introducción: Este es un trabajo de investigación que se centra en las elecciones generales españolas de 2015 y en la nueva celebración de los comicios en 2016, ya que en las primeras no hubo acuerdos suficientes para la formación de un ejecutivo estable. Durante este periodo, y especialmente desde 2015, la política española superó por primera vez en el reciente periodo democrático un bipartidismo histórico, anclado en el Partido Popular (PP) y el Partidos Socialista Obrero Español (PSOE), para dar paso a nuevas formaciones con opciones de gobierno nacional como fueron Podemos y Ciudadanos. Los principales elementos de la investigación conectan con los estudios de agendasetting, en donde se ofrece un análisis de la agenda pública española y la agenda mediática, deteniéndose en la cobertura política. Metodología: Se ha realizado un análisis de contenido en prensa de referencia (El Mundo y El País) durante los días de campaña de las dos elecciones mencionadas que permiten visibilizar los temas más importantes de agenda. Resultados y discusión: Los datos ofrecidos por el estudio reflejan que los problemas que los españoles señalan como importantes para el país se ven relegados a un segundo plano durante la campaña electoral. Los líderes y las diferentes formaciones prefieren visibilizar en sus discursos temas ligados a la política y la gobernabilidad, denominados "meta-temas", centrados en los pactos, acuerdos y automenciones frente a los temas clásicos de agenda pública como el empleo, la sanidad o la educación entre otros. 
PALABRAS CLAVE: elecciones 2015-2016; agenda; debate; gobernabilidad; bipartidismo; España.

\begin{abstract}
Introduction: This is a research paper that focuses on the Spanish General Elections of 2015 and the subsequent elections, which were held in 2016 as a result of the former ones not producing any agreements leading to the creation of a stable executive. During that period and, in particular, since 2015, politics in Spain overcame a historical two-party domination for the first time in its democracy - on the one hand, the Popular Party (PP) and the Spanish Socialist Workers' Party (PSOE) - giving way to new political parties aspiring to reach national government, for example, Podemos and Ciudadanos. The main elements of the research deal with studies in agenda setting including an analysis of the Spanish public and media agendas, with a particular emphasis on political coverage. Methodology: A content analysis of the press has been carried out (El Mundo and El Pais) during the campaign period of the aforementioned elections, revealing the most significant topics on the agenda. Results and Discussion: The data produced by the study indicate that the problems deemed important for the country by Spanish voters have been relegated to a second tier during the electoral campaign. Leaders and different groups prefer to highlight issues linked to politics and governance called "meta-topics" in their speeches, centering on pacts, agreements and self-reference rather than the usual issues of the political agenda such as employment, health and education, among others.
\end{abstract}

KEYWORDS: 2015 and 2016 elections; agenda, debate; governance; two-party system; Spain.

\title{
CONTENIDOS
}

1. Introducción: La ruptura del bipartidismo. 1.1. Sistema de partidos. 1.2. El bipartidismo "salta por los aires". 1.3. Ciudadanos y Podemos: la regeneración con diferentes matices. 1.4. Redoblada batalla por el electorado. 1.5. El problema de la gobernabilidad: el camino hacia 2016. 1.6. La política como tema de debate (meta-temas). 2. Marco teórico. La construcción de la agenda. 3. Metodología y temas de agenda. 4. Análisis y resultados: agendas en campaña electoral. 5. Discusión y conclusiones. 6. Referencias bibliográficas.

\section{Introducción: La ruptura del bipartidismo}

Las elecciones generales celebradas en 2015 y 2016, en la medida en que suponen la entrada de dos nuevos partidos -Podemos y Ciudadanos-, nos ofrecen una magnífica oportunidad para estudiar la construcción de la agenda de temas que cada fuerza política destaca en su discurso público. No sólo la de los nuevos actores, también la de los antiguos, que se ven afectados por la presencia de los recién llegados. Hemos considerado importante para una mejor comprensión del estudio explicar pormenorizadamente, a modo de contexto, cómo se desarrollaron las elecciones generales de $2015 \mathrm{y}$ el relato de cómo fue la negociación frustrada para la gobernabilidad que terminó en repetición de elecciones en 2016.

En las páginas que siguen analizaremos la irrupción de Podemos y Ciudadanos a través del análisis de uno de los componentes más importantes a la hora de construir una agenda de partido: los temas de debate que se priorizan. Porque a nadie se le escapa que la actividad política es una cuestión, en cierta medida, de énfasis: no transmite la misma sensación a la ciudadanía un partido o un candidato que incluye entre sus prioridades de gobierno la reivindicación de la unidad de España, por ejemplo, que otro que hace lo propio con la regeneración democrática, la justicia social, la inmigración o la economía. Pongamos algunos ejemplos: al Partido Popular se le ha asociado tradicionalmente con la 
buena gestión en materia económica o con el rigor presupuestario. A los socialistas con la justicia social moderada y la igualación de derechos de las minorías.

El marketing político, en cuanto a herramienta al servicio de políticos y candidatos (Maareck, 1997, p. 19), constituye un tipo de comunicación muy sensible a la realidad político-social en la que se aplica. Debe adaptarse al cambio social, modelando su discurso a las preferencias y tendencias más importantes del electorado, siempre con la mirada puesta en la idea o imagen que se desea construir a través de la priorización de temas. En esta dirección, presentamos un trabajo empírico, fundamentado en el análisis de dos elecciones generales españolas con un estudio de campo en el que se observan los principales temas o problemas de los españoles y su vinculación con el discurso político, difundido a través de la prensa de referencia del país, bajo los marcos conceptuales de la teoría de la agenda-setting.

\subsection{Sistema de partidos}

Las pautas de interrelación que cada una de las formaciones de un sistema establece con el resto de partidos son importantes; las dinámicas de competición partidista importan a la hora de construir la imagen de un grupo político.

La quiebra del sistema de partidos de corte bipartidista español nos ofrece un ejemplo muy claro. Los principales ejes temáticos ligados al PP y el PSOE -que habían permanecido más o menos estables desde 1982- de repente se ven desafiados por la irrupción, en las elecciones de 2015, de dos nuevas formaciones con vocación de gobierno. Podemos y Ciudadanos implican, sobre todo, una amenaza a la "vieja política" de populares y socialistas. Y éstos se ven obligados a redefinirlos o transformarlos, al considerarse amenazados.

De este modo, las elecciones de 2015 y 2016 son elecciones que introducen un cambio muy profundo en la forma de hacer y demandar política. Nada tienen que ver con consultas precedentes: las de 2011 o elecciones de la crisis económica (Colino y Cotarelo, 2012) o las de 2008 o elecciones de la crispación política (Balaguer y Sanz, 2010).

\subsection{EI bipartidismo "salta por los aires"}

Desde el realineamiento de 1982 (Caciagli, 1984; Gunther, 1986), el sistema de partidos español había estado marcado por lo que podríamos denominar un "bipartidismo imperfecto", en la medida en que parece cumplirse la máxima popularizada por Sartori (2005) de que la existencia de terceros partidos "no impide que los partidos principales gobiernen solos, esto es, cuando las coaliciones resultan innecesarias" (Ibíd. 240).

En el sistema bipartidista español se situaba en el centro izquierda el Partido Socialista Obrero Español (PSOE) y, en el centro derecha, Alianza Popular (AP) primero y, a partir de 1989, el actual Partido Popular (PP). La coletilla de "imperfecto" que puede añadírsele al sistema previo a 2015 tiene que ver con la existencia de los denominados "partidos bisagra". Esto es, pequeñas formaciones, generalmente nacionalistas, de las cuales, en ausencia de mayoría absoluta, ha dependido la investidura del presidente del Gobierno o, cuanto menos, la aprobación de las leyes más importantes de la legislatura o la propia estabilidad de los gobiernos.

De este modo, el bipartidismo español se ha caracterizado por la existencia de dos grandes formaciones que se han ido repartiendo las labores de gobierno. Pero, también, por toda una constelación de partidos más pequeños que, en ocasiones, han desempeñado un papel de árbitro muy 
relevante (Reniu, 2013). Estas dinámicas, que habían permanecido inalteradas durante más de treinta años, sufren una mutación súbita tras las elecciones generales de 2015.

\subsection{Ciudadanos y Podemos: la regeneración con diferentes matices}

Ciudadanos se sitúa, ideológicamente hablando, a la izquierda del PP y a la derecha del PSOE. Es decir, en un lugar en el que estaría en condiciones de recoger voto descontento de ambas formaciones. Sus principales propuestas diferenciales de la "política tradicional" tienen que ver con dos ideas fuerza: regeneración democrática y reivindicación de España.

El regeneracionismo de Ciudadanos cobra especial importancia en 2015 y 2016, para perder fuerza tras la llegada al poder del PSOE tras la moción de censura a Mariano Rajoy de junio de 2018. Pero se trata de una búsqueda de regeneración (motivada por la corrupción) desde una visión ordenada y moderada de la política. Este matiz busca diferenciarse de la marca y escenificación de Podemos, el otro nuevo actor que, al menos en esta dimensión, entra en competencia con el partido de Albert Rivera. Las elecciones del 2015 pueden entenderse, como una batalla por canalizar el voto descontento con el bipartidismo.

Como hemos dicho ya, al encontrarse los partidos interconectados dentro de un sistema, los ejes fundacionales de Ciudadanos impactan en el resto de formaciones. La regeneración señala hacia una "vieja política", vista como corrupta y agotada. PP y PSOE deben darse por aludidos. Pero esta crítica busca también tomar distancia con el reformismo que surge a la izquierda del PSOE, tachado por Ciudadanos como "populismo radical" de izquierdas. Esta podría ser, a grandes rasgos, la imagen que -al menos en 2015 y 2016- intentó construir Ciudadanos.

Si Ciudadanos, de alguna manera, elude o pasa de puntillas por su componente ideológico ${ }^{1}$, Podemos, el otro gran partido que irrumpe a escala nacional en 2015, es percibido como un partido ideológicamente mucho más marcado. En este caso, en posiciones progresistas muy claras; esto es, a la izquierda de los socialistas, en el lugar que tradicionalmente había ocupado Izquierda Unida (IU). Sin embargo, la estrategia populista puesta en marcha por la formación liderada, por aquel entonces, por la pareja formada por Pablo Iglesias e Íñigo Errejón, podría explicar, al menos en parte, su condición de contrincante del PSOE por el voto moderado de izquierda o, incluso, de centro.

Podemos no se limita a desempeñar el papel de una "nueva" IU. Pretende ofrecer otra imagen diferente, más ambiciosa. En este empeño, decide abandonar el viejo discurso y estrategias comunicativas de la izquierda post comunista, dejando atrás las apelaciones a la clase trabajadora y al cleavage de clase social (Lipset y Rokkan, 2001). En su lugar, pone en circulación un nuevo antagonismo: la "casta" frente a la "gente". Los de "arriba", los poderosos frente al ciudadano común.

De este modo, los posibles votantes de Podemos no se limitan ya a la clase trabajadora, apelación tradicional de la izquierda. Porque la "gente" está, efectivamente, compuesta por trabajadores; pero también por clases medias, trabajadores de la administración pública, desempleados, jubilados o amas de casa. Es la "gente de bien" frente a los poderosos, la casta política corrupta, los poderes fácticos económicos.

A este electorado ampliado al que aspira la política populista se le une el componente regeneracionista que ya comentábamos para el caso de Ciudadanos. La gente, los de "abajo", los

\footnotetext{
1 En algunas ocasiones han hablado de su componente "liberal", etiqueta ideológica que no forma parte de la tradición española, mucho más acostumbrada al eje izquierda-derecha.
} 
comunes... frente a la élite política de la "vieja política" bipartidista, sólo pendiente de sus propios intereses y de los de los poderosos: la banca, el Ibex 35, la omnipresente y conservadora Alemania de Ángela Merkel.

\subsection{Redoblada batalla por el electorado}

La marca "regeneracionismo" de Ciudadanos y Podemos -con los matices pro orden y anti poderosos que acabamos de analizar- explica, al menos en parte, la redoblada competencia electoral de 2015 y 2016. Todos aquellos ciudadanos que, según los barómetros del Centro de Investigaciones Sociológicas (CIS), consideraban a la clase política como una de sus principales preocupaciones, han podido encontrar en estas dos nuevas formaciones una válvula de escape.

Ciudadanos y Podemos, en este sentido, habrían actuado como una especie de amortiguadores de la desafección, permitiendo seguir participando a aquellos sectores sociales descontentos con la actuación del bipartidismo durante la crisis económica.

La crítica a los excesos del bipartidismo y a la corrupción permiten la disputa de los espacios aledaños al centro político, cosa que no deja de implicar, por lo menos, dos consecuencias que merece la pena destacar aquí: aumento de la competencia y una mayor dificultad en la formación de los gobiernos.

La primera de estas dos cuestiones parece clara. No es lo mismo que el centro esté competido por dos actores (PP y PSOE) y que los otros jugadores renuncien a él, quedándose en posiciones más extremas (Ciudadanos a la derecha y Podemos a la izquierda), a que estos cuatro actores -cada uno a su manera- intenten expandir sus apoyos electorales colisionando con los otros integrantes del sistema.

A mayor competencia, mayores posibilidades de conflicto, cuando un espacio ideológico y, sobre todo, un amplio nicho de votantes situados en él, entra dentro de las aspiraciones de más de un partido o candidato comienza la batalla. En esta tesitura es probable que los incentivos para la puesta en marcha de una comunicación política de corte negativa aumenten, tal y como se ha descrito en trabajos precedentes (Castromil, 2012).

Esta circunstancia suele llevar emparejada la sensación de que los sistemas político y mediático se encuentran más polarizados, con más lucha, menos posibilidad de acuerdo y más bronca. Cuando, en realidad, si entendemos polarización como la distancia entre los dos partidos ideológicamente más distantes, ésta ha descendido. Se podría expresar que, a mayor moderación, menor polarización política y mayor posibilidad de negativismo mediático.

Por este motivo, a los candidatos les resulta imprescindible la inclusión de atributos (McCombs, 2006) que clarifiquen ciertas diferencias entre ellos. Más que nada, para que el elector vea reducida la incertidumbre en la que podría estar inmerso: donde antes había dos, ahora se cuentan cuatro aspirantes a su apoyo electoral, algunos de ellos recién llegados.

\subsection{El problema de la gobernabilidad: el camino hacia 2016}

Con los nuevos actores ya en la Carrera de San Jerónimo de Madrid tras las elecciones de 2015, se inicia la XI Legislatura, con escasos meses de funcionamiento. El motivo se encuentra en la desconfianza mutua que provoca una disputa del centro político. Pactar con un partido que aspira a sustituirte, a destruirte -electoralmente- si puede, resulta mucho más difícil que pactar con alguien 
complementario a tu propio proyecto. Un gobierno de centro-derecha y derecha (o de centroizquierda e izquierda) parece más viable que otro formado por dos o más partidos de diferentes "centros": centro-derecha + centro; centro-izquierda + centro; centro-derecha + centro-izquierda.

La legislatura iniciada tras las elecciones del 20 de diciembre de 2015 duró sólo 188 días. El primer síntoma de atasco en la formación de gobierno se puede observar ya con la renuncia a formar gobierno del cabeza de lista del partido más votado en aquellas elecciones, Mariano Rajoy, del Partido Popular. El presidente en funciones sabía que no contaba con los apoyos suficientes, que el Parlamento había dejado de ser un lugar plácido para convertirse en la institución que regiría la vida política del país de ahora en adelante. De la primacía del ejecutivo sobre el legislativo que suponía el bipartidismo se pasa ahora a la preeminencia del legislativo sobre el ejecutivo.

El único intento serio, sometido a votación en los primeros días de marzo, fue el encabezado por el líder socialista, Pedro Sánchez, apoyado por Albert Rivera, de Ciudadanos. El rechazo de Podemos volvió imposible la investidura y convirtió a Sánchez en el primer candidato a presidente del Gobierno rechazado por la Cámara baja. La situación de bloqueo terminó por enquistarse, haciéndose necesaria la convocatoria de elecciones para el mes de junio de 2016. La convocatoria podría entenderse como una nueva oportunidad para los ciudadanos para confirmar la ruptura del sistema de partidos de 2015 o la vuelta al bipartidismo.

A pesar de la situación de bloqueo institucional surgida por el rechazo del Parlamento al pacto PSOE-Ciudadanos y a la incapacidad de Mariano Rajoy para encabezar un gobierno, las cosas no cambian demasiado tras las elecciones de 2016.

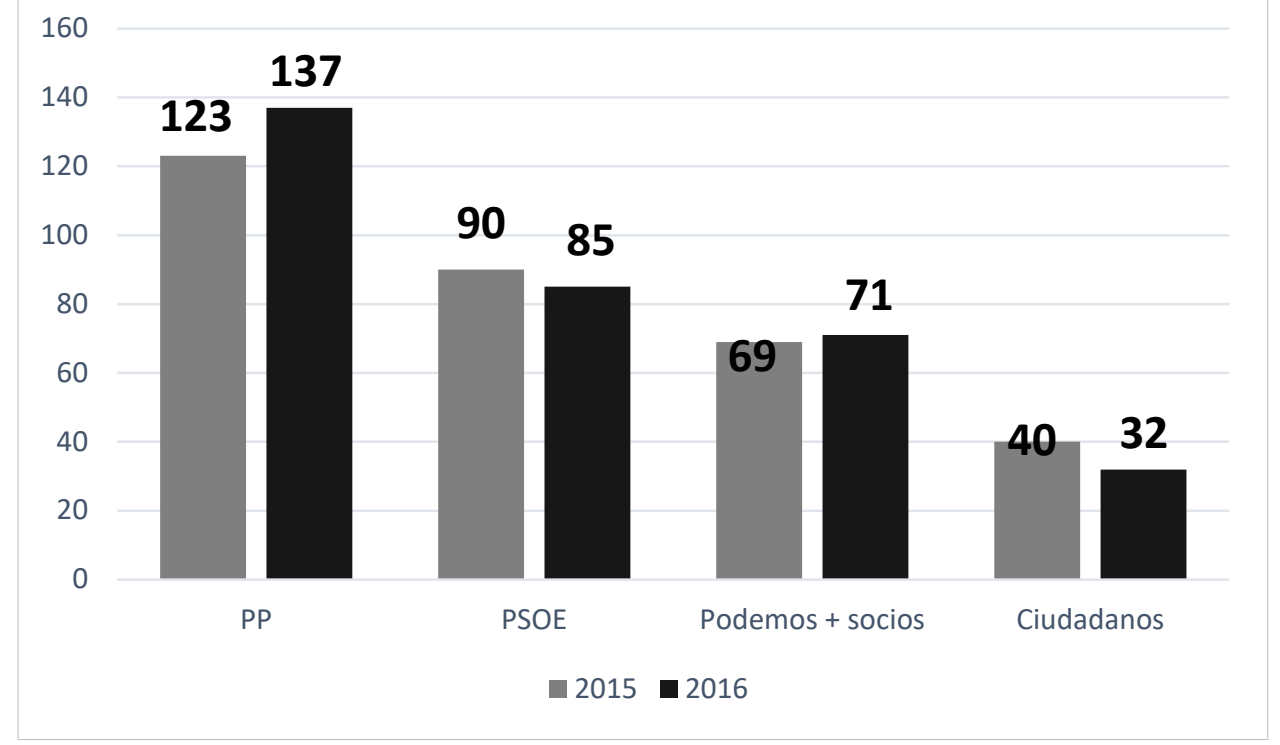

Gráfico 1. Evolución y reparto de escaños de los cuatro principales partidos (2015-2016). Fuente: Ministerio del Interior.

Los intentos frustrados de formar gobierno, que podrían haber hecho volver al redil del PP y del PSOE a muchos votantes descontentos, no parecen "apagar" las ansias de cambio de la sociedad española. Más bien, 2016 confirma, en lo esencial, lo ya conocido en 2015: el bipartidismo es sustituido por una nueva dinámica más plural y competitiva que incluye cuatro actores destacados: PP, PSOE, Podemos y Ciudadanos. 


\subsection{La política como tema de debate (meta-temas)}

Si, como acabamos de ver, las elecciones de 2016 pueden entenderse como consecuencia de las "contraindicaciones" de la política de pluralidad ampliada surgida de 2015 (ingobernabilidad); parece lógico que la campaña electoral de 2016 reflejase, de un modo u otro, esta circunstancia.

Se trata de una campaña en la que los temas de debate se vuelven autorreferenciales a la propia política, como veremos en el estudio empírico un poco más adelante. La cobertura de los principales medios de comunicación refleja, sobre todo, la posibilidad o no de un gobierno en solitario, el posible sorpasso de Podemos al PSOE o los pactos más factibles para la formación de gobierno.

Las elecciones de 2016 pueden entenderse como una consulta más bien atípica. En estos comicios, temas de debate clásicos como el paro, los problemas económicos o la inmigración se sitúan en un segundo plano frente a lo que hemos convenido en denominar meta-temas o temas de debate que tienen que ver con los cálculos estratégicos de los partidos para la formación de un gobierno estable.

\section{Marco teórico. La construcción de la agenda}

En el punto anterior hemos desarrollado el relato de qué ocurrió durante las elecciones 2015 y 2016 en donde los cuatro principales partidos se disputaban la gobernabilidad del país. Como en cualquier proceso democrático, esta realidad política pasó a los escenarios mediáticos en donde los ciudadanos son observadores de sus gestores públicos, a priori "expectantes" por conocer las iniciativas de debate para dar solución a los problemas de los españoles.

Los medios de comunicación reproducen y abordan los contenidos informativos ofreciendo a sus audiencias una realidad mediatizada. Como señala Lippmann (2003), se convierten en realidades de segunda mano en donde los ciudadanos no pueden acceder, en su mayoría, de manera directa ni a través de la experiencia personal al discurso de las formaciones políticas, pero sí a su mensaje mediatizado. En este sentido, Manin (1998) sostiene que la representación en las democracias contemporáneas está en proceso de cambio, de democracia de partidos a democracia de audiencias, con ello el autor resalta la importancia de los medios en la propia construcción de realidades.

La audiencia, entendida en este trabajo como potencial votante, hace uso de la prensa para informarse, observando la actuación política en relación con los temas de actualidad, especialmente los que resultan más relevantes en cuanto a cobertura informativa nacional. De manera que, tal y como sostienen los postulados de agenda-setting (McCombs, 2006), los medios generan marcos cognitivos que orientan a la gente a la hora de decidir cuáles son los problemas relevantes en función de la propuesta de temas que integra la agenda mediática a través sus contenidos.

Parafraseando las palabras de Cohen (1963), la prensa no tiene mucho éxito en decirnos qué pensar de un tema o partido, pero sí lo tiene en establecer cuáles son esos temas sobre los que pensar, asociados (mediáticamente) a la visibilidad temática del mensaje de las formaciones políticas. De manera que, paralelamente, en este sentido los debates mediáticos y especialmente los televisivos celebrados durante las campañas contribuyen a enfocar los temas de los discursos de los candidatos y sus formaciones (Conde-Vázquez, Fontenla-Pedreira y Rúas-Araújo, 2019).

Las investigaciones de agenda-setting replican en numerosos trabajos que existe una correlación muy alta entre los temas destacados por los medios, compilados en la agenda mediática, y los que lo son en la agenda pública, entendida esta última como los principales problemas del país o de un contexto objeto de estudio. Carballo, López-Escobar y McCombs (2018) exponen que la teoría de la agenda- 
setting tiene su pilar en el análisis de los medios de comunicación como elemento de influencia sobre la opinión de los ciudadanos y sus percepciones de la realidad.

Esta teoría plantea una función de relación entre el contenido de la agenda mediática y la agenda pública, el éxito de esta relación resulta de la búsqueda de orientación informativa de la opinión pública. Weaver (1980) expuso que esa necesidad de orientación de los individuos tiene su germen en factores psicológicos que hacen que las personas requieran y busquen contenidos que les permitan estar familiarizados con sus entornos. La política expresa un ejemplo de esta situación, resulta difícil obtener un conocimiento directo de los candidatos, siendo lo habitual aproximarse a ellos y a sus propuestas a través de los medios de comunicación. Siguiendo esta línea, es la prensa quien prioriza y selecciona del mensaje político aquellos temas que ocuparán informativamente las páginas de un medio. Esta práctica habitual modifica la comunicación política con estrategias de marketing que persiguen el posicionamiento del argumentario partidista en la prensa. Las formaciones han visto en los medios el lugar de su escenario político, en el que reafirmamos el concepto de Manin (1998). Esto es, se sostiene un cambio en las estrategias de comunicación política, pasando de democracia de partidos a democracia de audiencias.

Desde otra perspectiva, existen investigaciones centradas en el paralelismo político y mediático (Hallin y Mancini, 2004) que, para el caso español, desvirtúan el sistema democrático, ya que existe una vinculación entre grupos mediáticos y parlamentarios (González, Rodríguez y Castromil, 2010). González (2008) mantiene que los políticos buscan más el favor de la prensa trabajando en mayor medida para los medios que para los electores o la sociedad civil.

De manera que diremos que la agenda mediática no sólo es el conjunto de temas que la prensa señala como relevantes o como la actualidad más destacada del momento o país, otorgando mayor visibilidad o duración en todo tipo medios. También incorpora los temas que la propia opinión pública y la sociedad civil demandan, al igual que recoge las iniciativas parlamentarias (Wanta y Alkazemi, 2017).

La agenda pública está formada por el conjunto de menciones que responden al problema más importante de una sociedad, comunidad o país que establece desde la percepción (remarcamos percepción, porque esto es lo que mide y no la realidad) los temas más candentes para la opinión pública, demandando a su clase política soluciones. Los estudios tradicionales de agenda se basan en el estilo de pregunta introducido por Gallup ${ }^{2}$ expresado de la siguiente manera: "Dígame, ¿cuál es el problema que usted considera más importante para el país?". Emplearemos una formulación similar de la pregunta, la reproducida por el Centro de Investigaciones Sociológicas (CIS) que servirá como fuente para el seguimiento de agenda pública a través de los barómetros mensuales a lo largo de varios años.

Desde una perspectiva próxima a la propuesta de este artículo, centrada en el sistema español, son muchas y variadas las investigaciones que han observado la irrupción de los nuevos partidos en las agendas (Bosch y Durán, 2019; Lancaster, 2017). Para el seguimiento de las elecciones del 2015 y el 2016 existen publicaciones que han incorporado variados elementos para el análisis, como puedan ser el seguimiento de las redes sociales (Campos-Domínguez y Calvo, 2017; CaseroRipollés, Feenstra y Tormey, 2016), el análisis de género (Ferrín, Fraile y García-Albacete, 2019) o la representación televisiva de los políticos (García de Madaraiaga, Navarro y Olmo, 2019) entre otros.

\footnotetext{
${ }^{2}$ Gallup es una empresa estadounidense, fundada en 1935 por George Gallup, ligada en sus orígenes a la realización de encuestas de opinión pública.
} 


\section{Metodología y temas de agenda}

El objetivo principal de este trabajo pretende visibilizar cuáles son los temas más importantes de la agenda pública y su seguimiento en el mensaje de los partidos y líderes políticos españoles a través de los medios de comunicación. En este sentido, consideramos que la defensa o propuesta en mayor o menor medida de esos temas se convierte en una señal de identidad para las formaciones.

Esta señal de identidad potencia un tema en la estrategia de comunicación política y visibiliza unas propuestas frente a otras que movilizan más a unos votantes que a otros. La ruptura del sistema de partidos conlleva una mayor competencia política en la que la imagen de los partidos va ligada a temas con estrategias claras para adherir más electores, reforzando y modelando su ideología en función de la asociación con los temas.

Las elecciones generales del 20 diciembre 2015 supusieron una efervescencia informativa, política y ciudadana ya que era la primera vez en democracia que más de dos formaciones se repartían el electorado con posibilidades reales de gobernabilidad, rompiendo con ello el bipartidismo. Además de PP y PSOE, irrumpían en el arco parlamentario dos nuevos partidos que podían sumarse a la formación de gobierno: Podemos y Ciudadanos. Pese a ello, las negociaciones no fueron fructíferas y hubo que ir a unos nuevos comicios en junio 2016.

En esta dirección planteamos un análisis de los temas convencionales de agenda ligados a lo que hemos venido en denominar "meta-temas", entendidos como los temas políticos de debate vinculados a las estrategias de los pactos, la negociación y el propio debate entre las formaciones para llegar a un gobierno estable. El conjunto de issues analizados está compuesto por 15 elementos que son el resultado de los 10 primeros temas de agenda pública, según recoge el CIS durante el periodo objeto de estudio y expuestos en la tabla 1. A ellos se sumaron más temas de la propia actualidad informativa vinculados a la política nacional recogidos durante las dos semanas de campaña de ambas elecciones, algunos de ellos fueron la independencia de Cataluña o el cambio climático entre otros.

El trabajo que exponemos introduce dos preguntas de investigación: ¿Cuáles fueron los principales temas (meta-temas) durante la campaña electoral de 2015 y 2016? ¿Cuáles de esos temas (metatemas) iban ligados a cada uno de los cuatro partidos señalados?

Estas dos preguntas nos llevan a plantear la siguiente hipótesis: La ruptura del bipartidismo ha provocado que los prensa priorice en sus portadas los meta-temas del mensaje político en lugar de los temas clásicos de agenda, principalmente los establecidos por la agenda pública. Durante el periodo que ocupa esta investigación, los diarios destacan en primera plana la lucha, la contienda, los pactos o la negociación para la gobernabilidad dejando en un segundo lugar temas estructurales de la agenda como el paro, la economía, la sanidad, el terrorismo o la vivienda que en anteriores elecciones fueron los principales aspectos de visibilidad mediática, tal y como expresan numerosos trabajos que analizan la relevancia de los temas en los procesos electorales previos (Bouza y Rodríguez-Díaz, 2017; Chavero et al., 2013; Rodríguez-Díaz y Castromil, 2008).

Los problemas de la agenda pública son aquellos que se recogen de la pregunta de agenda del CIS enunciada como sigue: “¿Cuál es, a su juicio, el principal problema que existe actualmente en España? ¿Y el segundo? ¿Y el tercero? (RESPUESTA ESPONTANEA)”. De manera que los principales diez primeros problemas de los españoles (reflejados en los barómetros previos a los 
RLCS, Revista Latina de Comunicación Social, 76, 209-227

[Investigación] DOI: 10.4185/RLCS-2020-1444 | ISSN 1138-5820 | Año 2020

comicios) en el mes diciembre de $2015^{3}$ y junio de $2016^{4}$ fueron, por orden de importancia, los recogidos en la tabla 1.

Tabla 1. Agenda pública española.

\begin{tabular}{|c|c|c|c|c|}
\hline \multirow{2}{*}{$\begin{array}{c}\text { TEMA } \\
\text { Paro }\end{array}$} & \multicolumn{2}{|c|}{ POSICIÓN - diciembre 2015} & \multicolumn{2}{|c|}{ POSICIÓN - junio 2016} \\
\hline & $1^{\mathrm{a}}$ & $79,8 \%$ & $1^{\mathrm{a}}$ & $76,4 \%$ \\
\hline Corrupción y fraude & $2^{\mathrm{a}}$ & $38,8 \%$ & $2^{\mathrm{a}}$ & $44,9 \%$ \\
\hline Problemas económicos & $3^{\mathrm{a}}$ & $24,4 \%$ & $3^{\mathrm{a}}$ & $25,1 \%$ \\
\hline Políticos en general & $4^{a}$ & $14,8 \%$ & $4^{\mathrm{a}}$ & $21,1 \%$ \\
\hline Sanidad & $5^{\mathrm{a}}$ & $11,9 \%$ & $6^{\mathrm{a}}$ & $10 \%$ \\
\hline Problemas de índole social & $6^{\mathrm{a}}$ & $11,8 \%$ & $5^{\mathrm{a}}$ & $11,6 \%$ \\
\hline Educación & $7^{\mathrm{a}}$ & $9,7 \%$ & $7^{\mathrm{a}}$ & $9,7 \%$ \\
\hline Terrorismo Internacional & $8^{\circ}$ & $9 \%$ & & \\
\hline Calidad del empleo & $9^{a}$ & $3,9 \%$ & $8^{\mathrm{a}}$ & $4,8 \%$ \\
\hline Inmigración & $10^{\mathrm{a}}$ & $3,8 \%$ & $10^{\mathrm{a}}$ & $3,3 \%$ \\
\hline Falta de Gobierno & & & $8^{\mathrm{a}}$ & $4,8 \%$ \\
\hline
\end{tabular}

Fuente: CIS. Elaboración propia.

Los españoles, a través de la agenda pública, señalaron el paro, la corrupción y el fraude, los problemas económicos, los políticos, la sanidad y, entre otros, la "falta de gobierno", como los principales problemas del país, tanto en 2015 como en 2016. Se entiende con esto que los políticos deberían centrar su discurso y actuación en ellos por considerarse problemas prioritarios para los ciudadanos. A su vez, algunos de estos temas sirven de anclajes y estandartes para las nuevas formaciones políticas, tal y como hemos desarrollado al inicio de este artículo.

En paralelo, y para seguir la información política ligada a los temas y las formaciones a través de la agenda mediática, se ha realizado un análisis de contenido en dos diarios de referencia españoles con una línea editorial diferente (El Mundo y El País) durante las dos semanas previas de campaña electoral de los comicios celebrados el 20 de diciembre de 2015 y el 26 de junio de 2016, respectivamente. Para esta muestra, se ha contabilizado el número de menciones de cada uno de los quince temas señalados que estaban vinculados a la política nacional y/o a los cuatro principales partidos $^{5}$ en las portadas de ambos diarios en su edición impresa, obtenida de los PDF que ofrecen los periódicos. El número total de unidades de análisis han sido 176 titulares y piezas informativas de portada: 88 en el año 2015 (46 de El Mundo y 42 de El País) y 88 en el año 2016 (47 de El Mundo y 41 de El País).

Para el seguimiento de los temas partimos del análisis de los problemas propuestos por los españoles en la agenda pública y su presencia en la agenda mediática, vinculada especialmente a los temas políticos. A ello incorporamos otros conceptos políticos ligados a aspectos como puedan ser el desarrollo de la campaña electoral, la negociación política o gobernabilidad que hemos determinado como meta-temas, expresados y recogidos en la categoría de "partidos políticos". Principalmente aluden a las relaciones y diálogo entre los diferentes grupos para la formación de Gobierno, en un nuevo, muy nuevo escenario político fragmentado. Estos meta-temas, que representan el objeto de negociación de la nueva realidad parlamentaria no son ocasionales ni transitorios, sino que parece

\footnotetext{
${ }^{3}$ Estudio n ${ }^{\circ} 3121$, barómetro del CIS de diciembre de 2015.

${ }^{4}$ Estudio n⿳3 3142 , barómetro del CIS de junio de 2016.

${ }^{5}$ Partido Popular, PSOE, Podemos y Ciudadanos.
} 
que vienen para quedarse en el sistema político español y están vinculados a los temas ya mencionados como: gobierno, partidos políticos, regeneración, pactos, negociación, propuestas, decisiones y equipos entre otros.

\section{Análisis y resultados: agendas en campaña electoral}

En el marco introductorio se ha desarrollado cómo ha sido la ruptura del bipartidismo y en las siguientes líneas ofreceremos algunos datos que ayudarán a explicar cuáles fueron los temas más importantes que señalaron los españoles y cómo evolucionaron durante los procesos electorales estudiados.

Los resultados de los diferentes barómetros mensuales del CIS ofrecen una visión longitudinal de los problemas prioritarios para la opinión pública, conformando con ello la agenda pública. En el gráfico 2 se aprecia el conjunto de los seis primeros temas de preocupación desde diciembre de 2014 hasta mayo 2016, durante este periodo se celebraron los dos comicios electorales objeto de estudio en esta investigación.

Como se contempla en el gráfico 2, el paro o desempleo se convierte en el tema más destacado con mucha diferencia del resto de temas de agenda pública con porcentajes superiores al $75 \%$. En estos meses de estudio, la corrupción (entre el 60\% - 45\%) ocupa el segundo lugar con porcentajes dominantes junto a los problemas económicos $(25 \%)$. Este tercer problema contempla un aspecto muy amplio que aglutina a cualquier tipo de variable económica que determina la buena o mala marcha de los individuos; de manera que, junto al paro, los problemas económicos se convierten un eje vertebrador y de especial observación para la opinión pública. En un cuarto puesto estarían los políticos o la clase política en general (entre el 15\% - 25\%) con un repunte importante a partir de diciembre de 2015, momento de la celebración de las elecciones, no llegando a un acuerdo de gobernabilidad que provoca ir a nuevas elecciones en junio de 2016. Los problemas sociales y la sanidad (entre el 10\% - 14\%) compiten por las posiciones quita y sexta de la agenda ciudadana, con una relevancia muy inferior al resto de problemas que les preceden.

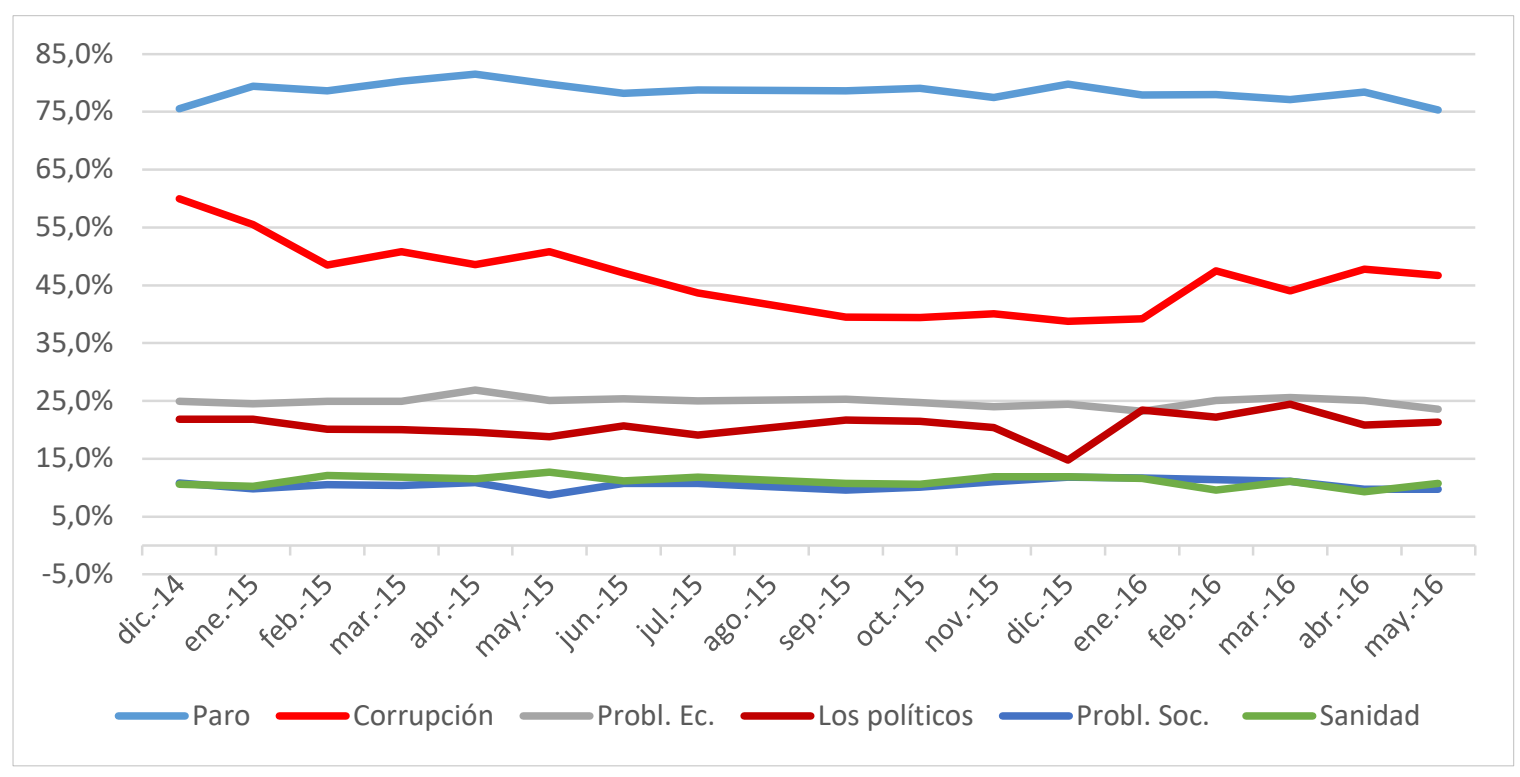

Gráfico 2. Evolución de los principales temas de la agenda pública 2014-2016.

Fuente: CIS. Elaboración propia. 
En los siguientes gráficos se visibilizan los resultados del análisis de contenido de los dos diarios durante las dos campañas electorales que tuvieron lugar del 4 al 18 de diciembre de 2015 y del 10 al 24 de junio de 2016, en donde los temas presentados en sus portadas se convierten en el hilo argumental del estudio. Se han contemplado para el análisis el seguimiento de los temas de la agenda pública, más los propios temas periodísticos o de actualidad informativa que durante los días señalados ocuparon las portadas de El Mundo y El País. El conjunto de estos temas suma un total de quince: Paro, Gobierno, partidos políticos, sanidad, educación, economía, "corrupción y fraude", recortes, juventud, inmigración, sociedad, terrorismo, Cataluña, Venezuela y Cambio Climático.

En los gráficos exponemos los resultados del análisis en donde diferenciamos entre temas mayores (aquellos que tienen más presencia diaria durante la campaña en los medios) y temas menores (aquellos que tienen menos presencia), aunque algunos de ellos podrían registrar variaciones dependiendo de si se trata de las elecciones del 2015 o del 2016.

Esta diferenciación entre temas mayores y menores estriba en que, pese a que ambos forman parte de las portadas de los periódicos, lo hacen con visibilidades diversas. Los temas mayores se posicionan en la primera página de manera constante, casi diaria, en ambas campañas en comparación con la irregularidad de los temas menores, con menos presencia en la agenda.

Los temas que conformaron la agenda mediática (gráfico 3) como temas mayores fueron: Gobierno, partidos políticos, economía, corrupción y fraude, terrorismo y Venezuela. Como temas menores: Paro, sanidad, educación, recortes, juventud e inmigración. Esta bifurcación entre mayores y menores pone en valor claramente la relevancia de los meta-temas (más políticos) frente a los de agenda pública, ocupando el lugar de los menores aquellos que mejor representan la agenda pública: paro, la sanidad, la educación, la economía, los recortes o sanidad.

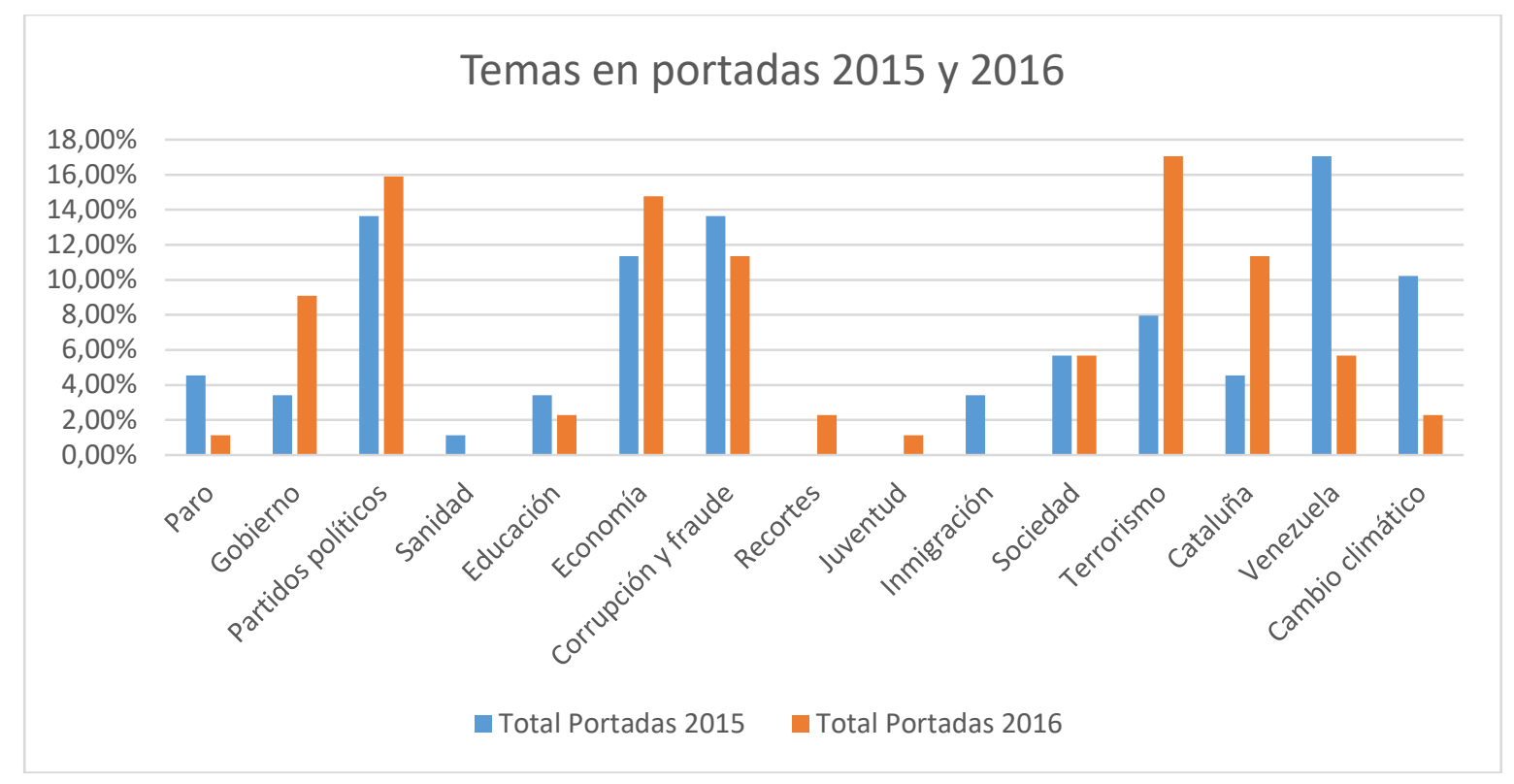

Gráfico 3. Principales temas en los diarios durante la campaña del 2015 y 2016.

Fuente: elaboración propia.

En el gráfico 3 se visualizan cuáles fueron los temas que tuvieron mayor cobertura informativa en ambas elecciones. Se aprecia que durante la campaña del 2015 los partidos políticos, la economía, la 
corrupción y el fraude y Venezuela ${ }^{6}$ fueron los principales temas en la agenda mediática con alusiones a la política española (a lo que sumaron los temas del cambio climático por celebrarse durante esas fechas una de las cumbres mundiales y el terrorismo, ligado a la actualidad internacional con menciones en clave nacional) ${ }^{7}$. De manera que sólo eran coincidentes con los temas mayores de la agenda pública: los partidos políticos, la corrupción y el fraude y la economía. En este sentido, contemplamos a la clase política como uno de los principales temas informativos cuando sus apariciones estaban ligadas a aspectos de regeneración, pactos y futura gobernabilidad que las formaciones mantenían en su discurso, especialmente introducidos en el nuevo escenario, dinamizado por la irrupción Podemos y Ciudadanos. El tema de la crisis en Venezuela se convirtió en un debate político de "desprestigio", con claras alusiones a la vinculación anterior de los líderes de Podemos con este país.

Sin embargo, durante la campaña de 2016 la cobertura informativa visibilizó un mayor aumento de los meta-temas con respecto a las elecciones de 2015, en donde la falta de acuerdo destacó la presencia de algunos de esos meta-temas expuestos en esta investigación, sumados a otro conflicto político como es la independencia de Cataluña.

Estos elementos, expuestos en el gráfico 4, reflejan que los principales temas de la agenda mediática fueron: los partidos políticos, la economía, la corrupción, el Gobierno y Cataluña. A lo que se incorporó el terrorismo como tema destacado, conectado a actos de terrorismo internacional acaecidos durante esos días. Tanto en el gráfico 4 como en el gráfico 5 se puede apreciar la cobertura de los periódicos El Mundo y El País durante las campañas electorales del 2015 y 2016 en sus portadas.

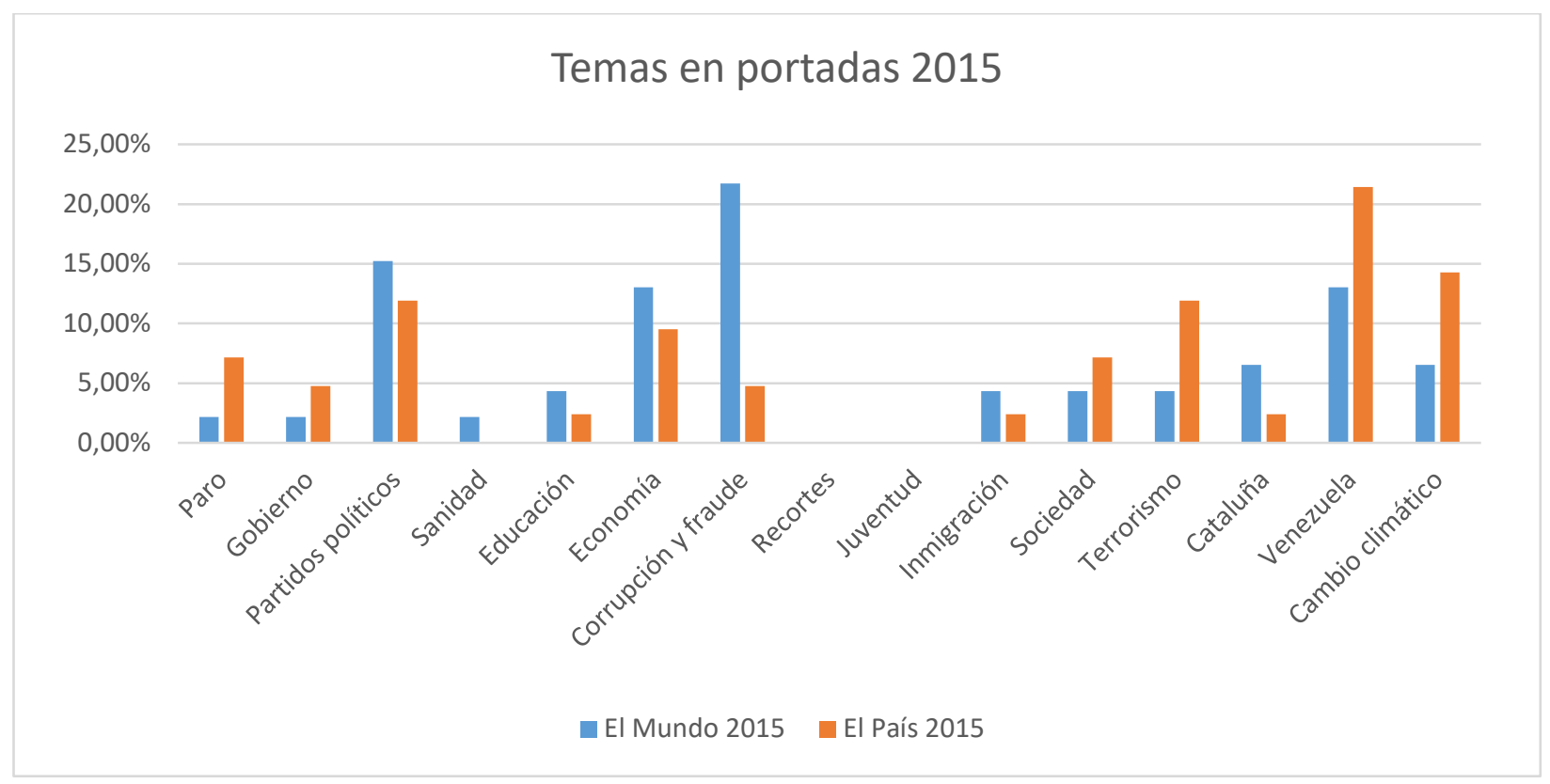

Gráfico 4. Principales temas por diario 2015.

Fuente: elaboración propia.

\footnotetext{
${ }^{6}$ El tema Venezuela no solo surge por la crisis del país vivida en el país americano, sino porque se asocia directamente con Podemos y sus relaciones previas con los líderes venezolanos.

7 Ambos temas fueron recogidos en la codificación por encontrarse presentes en la agenda pública española (en posiciones inferiores), pero se descartaron para el análisis de la investigación ya que sólo incidieron en la campaña política de manera transversal.
} 


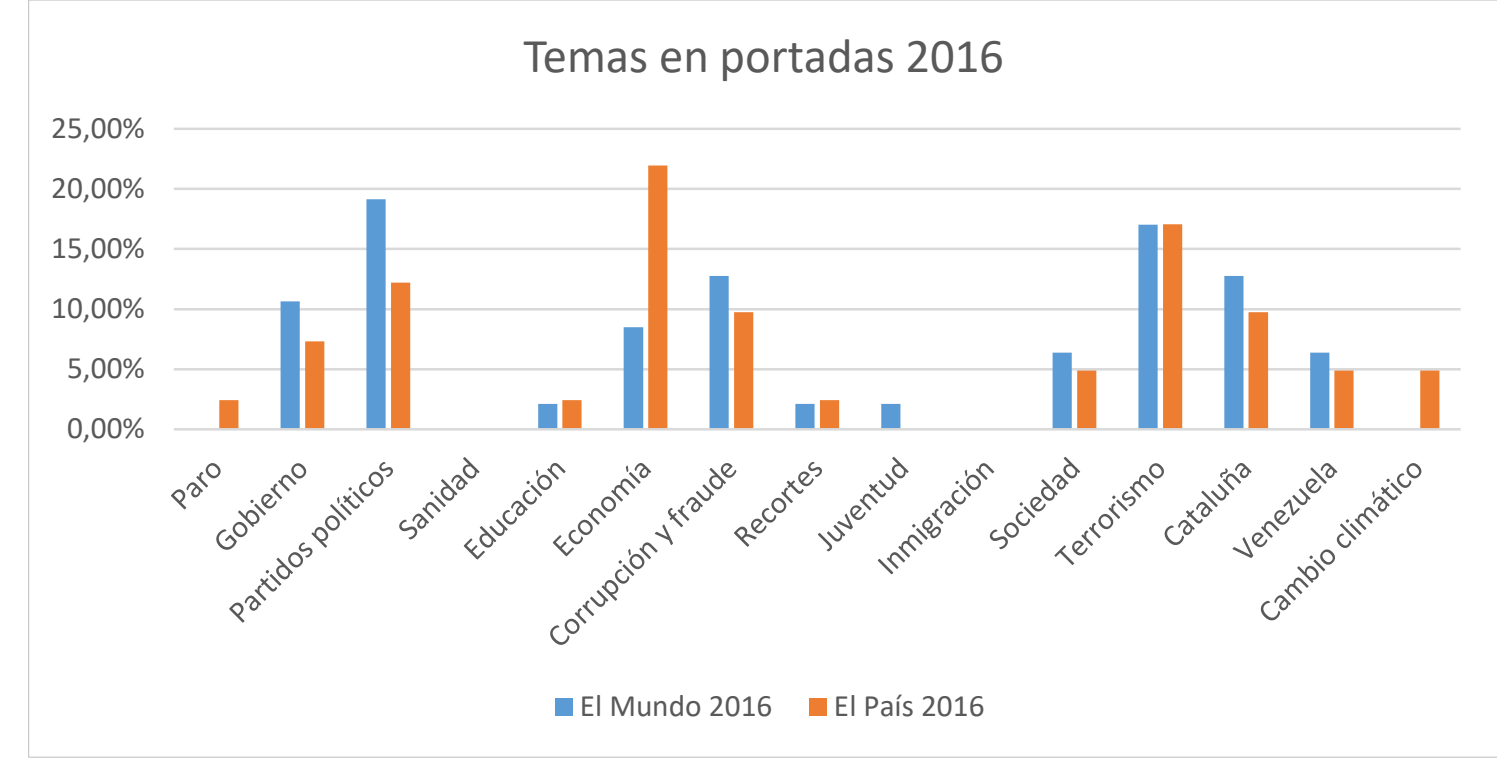

Gráfico 5. Principales temas por diario 2016.

Fuente: elaboración propia.

Desde una visión mediática y de línea editorial, en el gráfico 4 se aprecia cómo, durante la campaña electoral de las elecciones de 2015, ambos diarios destacaron principalmente los temas ligados al enfrentamiento y negociación política, junto a la economía y Venezuela. Pese a ello, El Mundo priorizó en su primera plana informaciones vinculadas a la corrupción y el fraude mientras que $E l$ País lo hizo con Venezuela, especialmente a través de la vinculación de Podemos con líderes venezolanos. Por otro lado, durante la campaña del 2016 (gráfico 5), temas como los partidos políticos, la economía, la corrupción y fraude y Cataluña ocuparon la mayor parte de las portadas diarias en ambos periódicos.

En una estrategia paralela a la presentada por la prensa, ofrecemos en los gráficos siguientes (gráficos 6 y 7) el análisis mediático de los temas ligados directamente a los partidos, en donde sólo han formado parte de la muestra las informaciones que mencionaban expresamente el nombre de los partidos y/o sus líderes. Con ello se aprecia cuál fue la asociación de cada tema con los cuatro partidos en función de su representación informativa. 
RLCS, Revista Latina de Comunicación Social, 76, 209-227

[Investigación] DOI: 10.4185/RLCS-2020-1444 | ISSN 1138-5820 | Año 2020

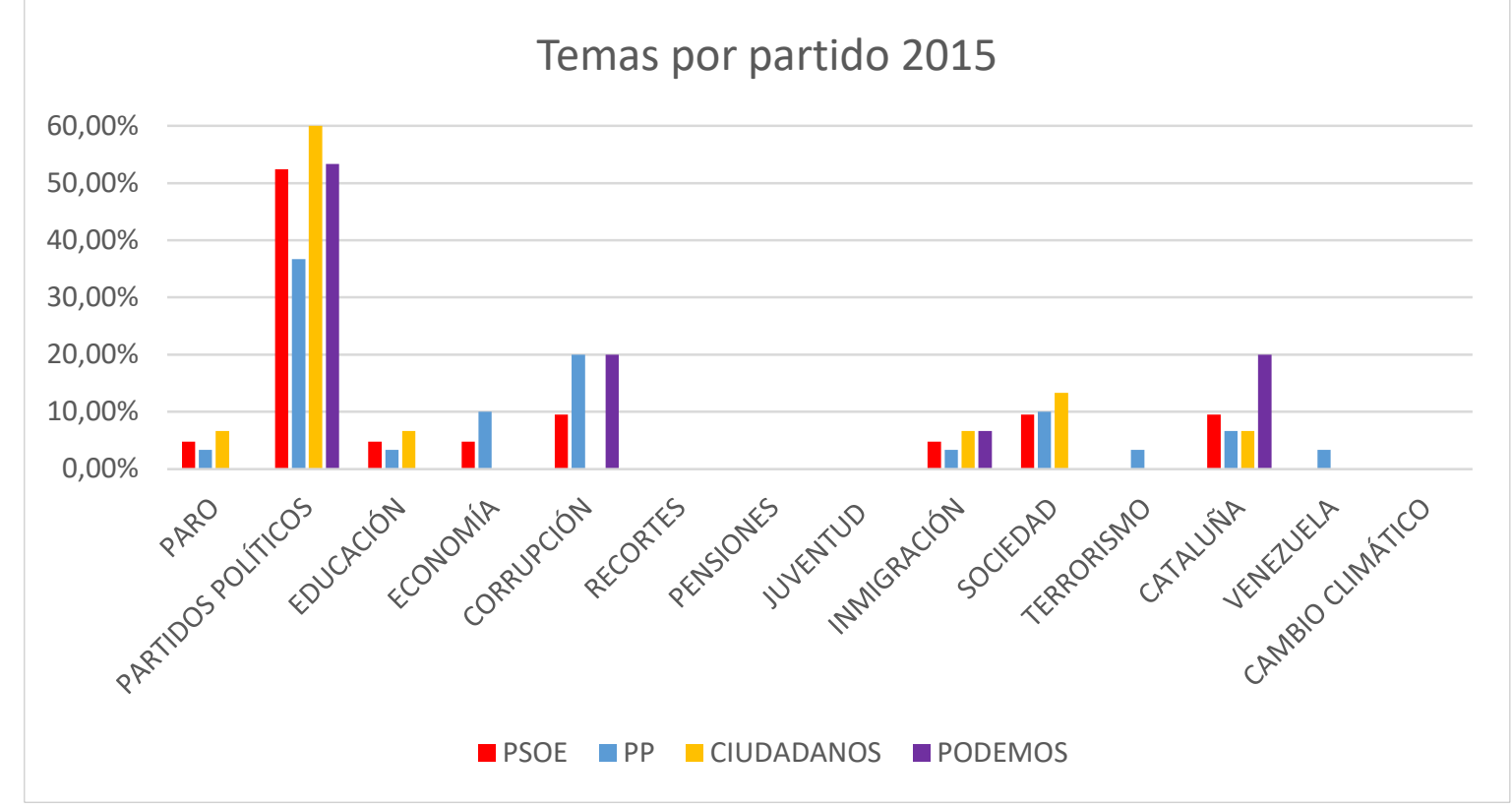

Gráfico 6. Principales temas por partido político 2015.

Fuente: elaboración propia.

Se observa como los temas ligados a partidos políticos (entendidos como meta-temas) se establecieron como elementos clave durante los dos procesos electorales, dominando sobre cualquier otra propuesta informativa. Durante la campaña de 2015 estuvo muy presente en los cuatro partidos, ocupando aproximadamente entre un 35\% (Partido Popular) y un 60\% (Ciudadanos) de los temas político nacionales recogidos en la prensa.

En las elecciones de 2015 (gráfico 5), y por orden de importancia, la corrupción y el fraude se posicionaron en un segundo plano en donde el Partido Popular y Podemos activaron este tema (por diferentes motivos) seguido de Cataluña, encabezado como problema ligado a las cuatro formaciones, especialmente el PSOE y Podemos.

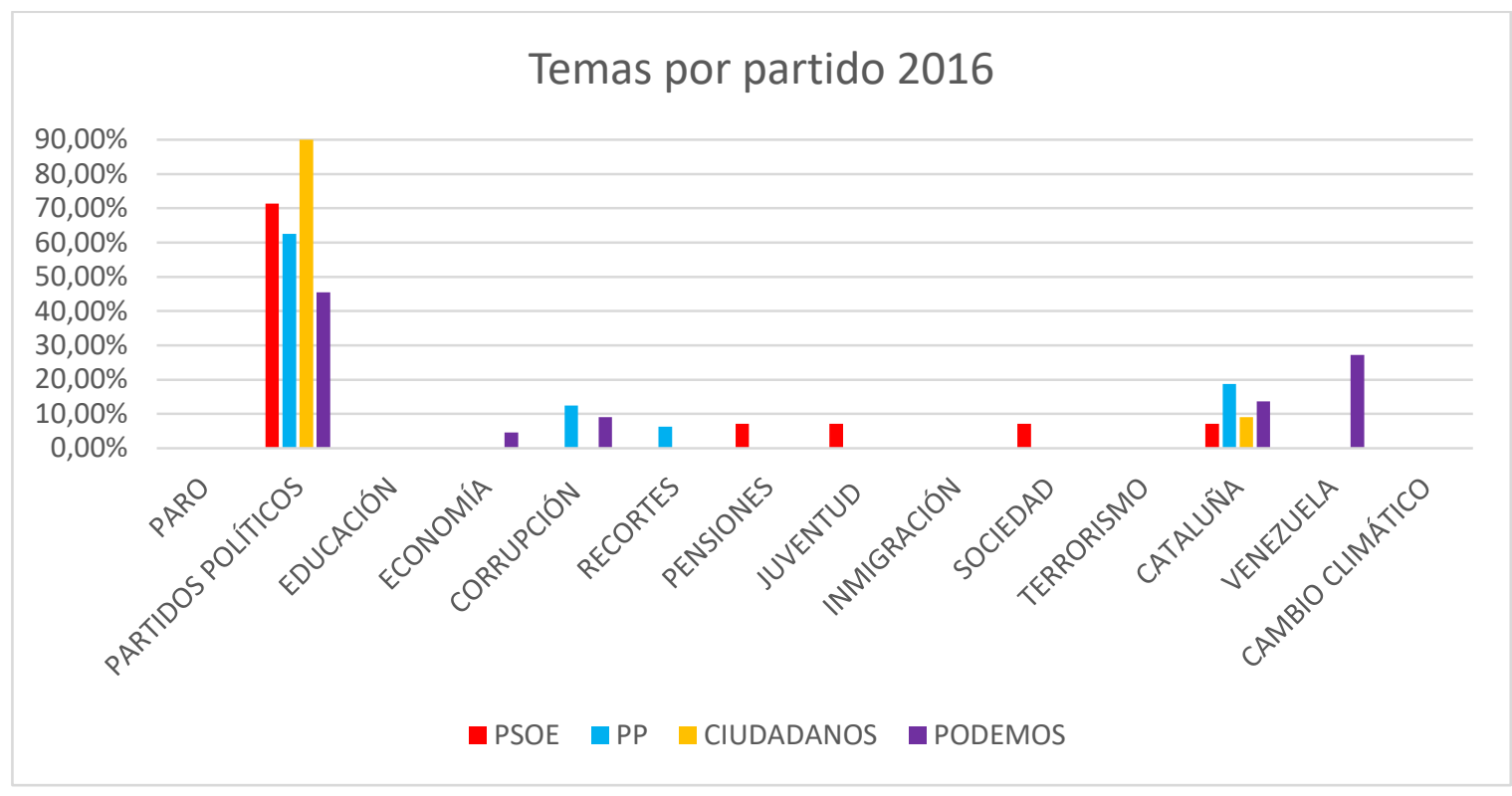

Gráfico 7. Principales temas por partido político 2016.

Fuente: elaboración propia. 
De la misma manera que en la campaña anterior, en la del 2016 todos los componentes de los metatemas irrumpen aún con más fuerza en donde esta categoría conglomerante denominada "partidos políticos" ocupa el eje de la campaña. Se aprecia en el gráfico 6 que la propia relación de los partidos, hablando de posibles pactos o negociaciones (entendidos como meta-temas más allá de las imperantes preocupaciones de los ciudadanos) continúa con mucha presencia en las elecciones del 2016, sumándose la independencia de Cataluña y Venezuela.

Para cualquiera de las formaciones presentadas, el tema se erigió como el estelar y clave para ellos y la gobernabilidad de España. La negociación, los pactos, la conveniencia de un debate y otros muchos elementos hicieron que el tema estuviera presente entre el 45\% (Podemos) y el 90\% (Ciudadanos) de las menciones que los periódicos realizaron cuando asociaron el discurso del partido con el tema.

\section{Discusión y conclusiones}

Tal y como presentamos al inicio de este estudio, los resultados evidencian que las principales temáticas de la agenda pública (entendidas como problemas de los españoles) no parecen ser los ejes más destacados para los políticos durante las dos campañas analizadas. Problemas estructurales por su importancia para los ciudadanos como son el paro, la corrupción y la economía no se corresponden en proporciones similares con la presencia del mensaje político en los medios, al menos en sus portadas.

Sin embargo, este trabajo evidencia que existe una primacía mediática hacia los temas políticos centrados en la confrontación y no tanto en los principales aspectos de la agenda pública. Los metatemas (partidos políticos, corrupción y fraude, gobernabilidad, negociación, gobierno...) han funcionado durante el periodo analizado como el eje vertebrador del mensaje de los partidos políticos a través de la prensa.

Con la ruptura del bipartidismo, los resultados, tanto de las elecciones del 2015 como las del 2016, confirman la hipótesis inicial del paso de los temas a los meta-temas. Los medios de comunicación priorizan el conflicto entre los líderes, la negociación y la búsqueda de acuerdos programáticos perdurables que funcionen en el tiempo. Con ello dejan en un segundo plano, como temas menores, a los elementos centrales de la agenda pública ya sean el paro, la precariedad en el empleo, la inmigración o la sanidad, entendidos como problemas que necesitan soluciones por parte de los grupos parlamentarios.

Los temas conectados con el concepto de regeneración democrática - crítica al bipartidismo planteado en las elecciones estudiadas, sumado a la crisis- precariedad económica y a la corrupción, parecen desvanecerse en un círculo vicioso en el que formar gobierno resulta complicado o imposible ya que los pactos y la negociación son difíciles de conseguir y, aún más, de mantener. A ello se añade el tema de Cataluña como identidad nacional, que supone otro de los escollos en la futura gestión de las decisiones políticas. Todo ello obliga a una constante negociación y fracaso de pactos que desbordan y posicionan el mensaje político y mediático a diferentes bandas, provocando en los españoles una desafección hacia su clase política, considerándola más un problema en sí misma que una gestora de las soluciones para el país, especialmente para resolver los temas de la agenda pública.

\section{Referencias bibliográficas}

Balaguer, J. y Sanz, A. (2010). La segunda ola de la crispación: competición y polarización en la VIII legislatura. En J. R. Montero, y I. Lago (Eds.): Elecciones Generales 2008 (pp. 61-92). Centro de Investigaciones Sociológicas (CIS). 
RLCS, Revista Latina de Comunicación Social, 76, 209-227

[Investigación] DOI: 10.4185/RLCS-2020-1444 | ISSN 1138-5820 | Año 2020

Bosch, A. \& Durán, I. M. (2019). How does economic crisis impel emerging parties on the road to elections? The case of the Spanish Podemos and Ciudadanos. Party Politics, 25(2), 257-267.

Bouza, F. y Rodríguez-Díaz, R. (2017). Área de impacto de la comunicación política: estudio de caso (elecciones españolas 2004). Sociologiados. Revista de investigación social, 2(1), 19-34. https://doi.org/10.14198/socdos.2017.1.02

Caciagli, M. (1984). España 1982: Las elecciones del cambio. Revista Española de Investigaciones Sociológicas, (28), 85-118. https://doi.org/10.2307/40183101

Campos-Domínguez, E. y Calvo, D. (2017). La campaña electoral en internet: planificación, repercusión y viralización en Twitter durante las elecciones españolas de 2015. Comunicación y sociedad, (29), 93-116. http://www.scielo.org.mx/pdf/comso/n29/0188-252X-comso-29-00093.pdf

Carballo, M., López-Escobar, E. y McCombs, M. (2018). Communication, Public Opinion, and Democracy: New Challenges. Communication \& Society, 31(4), 121-134.

https://doi.org/10.15581/003.31.4.121-133

Casero-Ripollés, A., Feenstra, R. y Tormey, S. (2016). Old and New Media Logics in an Electoral Campaign: The Case of Podemos and the Two-Way Street Mediatization of Politics. The International Journal of Press/Politics, 21(3), 378-397.

https://doi.org/10.1177/1940161216645340

Castromil, A. (2012). Negativismo mediático y campaña electoral en las elecciones generales de 2008. Revista Española de Investigaciones Sociológicas, (139), 163-174.

https://doi.org/10.5477/cis/reis.139.163

Chavero, P., González, J. J., Bouza, F., Castromil, A. R. y Rodríguez, R. (2013). La mediatización de la agenda política: la discusión del pacto social como conflicto de agendas, 2009-2011. Revista latina de comunicación social, (68), 639-655. https://www.redalyc.org/articulo.oa?id=81928785028

Cohen, B. (1963). The press and foreign policy. Princeton University Press.

Colino, C. y Cotarelo, R. (Comp.) (2012). España en crisis. Balance de la segunda legislatura de Rodríguez Zapatero. Tirant Humanidades.

Conde-Vázquez, E., Fontenla-Pedreira, J. y Rúas-Araújo, J. (Eds.) (2019). Debates electorales televisados: del antes al después. Cuadernos Artesanos de Comunicación, (154). Latina. https://doi.org/10.4185/cac154

Ferrín, M., Fraile, M. \& García-Albacete, G. M. (2019). Who Learns in Information Rich Contexts? The Informative Effects of the 2015 Spanish Electoral Campaign. The International Journal of Press/Politics. https://doi.org/10.1177/1940161219832455

García-de-Madariaga, J, M., Navarro, J. A. \& Olmo, A. (2019). A Mirage of Change: Visibility of Politicians on TVE during the 2015 and 2016 General Elections. Journalism Practice, 1-19. https://doi.org/10.1080/17512786.2019.1598885 
González, J. J. (2008). Los medios en la democracia avanzada. ¿Hacia una democracia de audiencia? En J. J. González y M. Requena, Tres Décadas de Cambo Social en España (pp. 345-382), segunda edición. Alianza Editorial.

González, J. J., Rodríguez, R. \& Castromil, A. R. (2010). A Case of Polarized Pluralism in a Mediterranean country. The Media and Politics in Spain. Global Media Journal: Mediterranean Edition, 5(1/2), 1-9. http://globalmedia.emu.edu.tr/images/stories/ALL_ARTICLES/2009 10 Fall Spring/Gonzalez $\underline{\text { Rodriguez Castromil.pdf }}$

Gunther, R. (1986). El realineamiento del sistema de partidos de 1982. En Linz, J. J. y Montero, J. R. (Ed.): Crisis y cambio: electores y partidos en la España de los años ochenta (pp. 27-69). Centro de Estudios Constitucionales.

Hallin, D. y Mancini, P. (2004). Comparing Media Systems: Three Models of Media and Politics. University Press.

Lancaster, T. D. (2017). The Spanish general elections of 2015 and 2016: a new stage in democratic politics. West European Politics, 40(4), 919-937. https://doi.org/10.1080/01402382.2017.1293340

Lippmann, W. (2003) [1922]. Opinión Pública. Langre.

Lipset, S. M. y Rokkan, S. (2001). Estructuras de división, sistemas de partidos y alineamientos electorales. En A. Batlle (Ed.): Diez textos básicos de ciencia política (pp. 231-273). Ariel.

Maareck, P. J. (1997). Marketing político y comunicación. Claves para una buena información política. Paidós.

Manin, B. (1998). Los principios del gobierno representativo. Alianza Editorial - Ciencias Sociales.

McCombs, M. (2006). Estableciendo la agenda. El impacto de los medios de comunicación en la opinión pública y el conocimiento. Paidós.

Reniu, J. Ma (Ed.) (2013). Pactar para gobernar. Dinámicas coalicionales en la España multinivel. Tirant lo Blanch.

Rodríguez, R. y Castromil, A. R. (2010). La circulación social de los encuadres periodísticos en tiempo de campaña electoral: Transmisión, influencia y atribución de responsabilidad. ZER: Revista de Estudios de Comunicación= Komunikazio Ikasketen Aldizkaria, 15(29), 193-212. https://www.ehu.eus/ojs/index.php/Zer/article/view/1652/1450

Wanta, W. \& Alkamezi, M. F. (2017). Agenda-setting: History and research tradition. The international encyclopedia of media effects, 1-14. https://doi.org/10.1002/9781118783764.wbieme0030

Weaver, D. (1980). Audience need for orientation and media effects. Communication Research, 7(3), 361-373. https://doi.org/10.1177/009365028000700305 


\title{
AUTORES:
}

\section{Raquel Rodríguez-Díaz}

Doctora y licenciada en Ciencias de la Información por la Universidad Complutense de Madrid y profesora Titular de Universidad del área de Periodismo en la Facultad de Ciencias de la Comunicación de la Universidad Rey Juan Carlos. Investiga sobre los efectos de los medios de comunicación, la comunicación política, la teoría del framing y la agenda setting a través de los temas de opinión pública española. Cuenta con varios sexenios de investigación y ha participado en proyectos de financiación pública y privada, nacionales e internacionales. Ha sido miembro del grupo de investigación Agenda y voto en la Facultad de Ciencias de la Información en la Universidad Complutense de Madrid.

raquel.rodriguez@urjc.es

Î́ndice H: 15

Orcid ID: https://orcid.org/0000-0002-8097-6585

Google Scholar: $\underline{\text { https://scholar.google.es/citations? user=n8HwtaIAAAAJ\&hl=es }}$

\author{
Antón R. Castromil \\ Doctor en Comunicación por la Universidad Complutense de Madrid (UCM), cuenta con estudios en \\ Comunicación y Periodismo en la Universidad Pontificia de Salamanca, Ciencias Políticas en la \\ UCM y Análisis de Datos de Encuesta en la University of Essex (Reino Unido) y es profesor del \\ Departamento de Sociología Aplicada de la UCM. Ha sido investigador visitante en la Universidad \\ de Cabo Verde, la Universidade Técnica de Lisboa (Portugal) y la University of Hull (Reino Unido). \\ Ha sido investigador de varios proyectos financiados por el Plan Nacional Español de I+D desde \\ 2008. Su investigación abarca la comunicación política, la opinión pública y los efectos de los \\ medios en los regímenes democráticos. Su trabajo en teoría de la comunicación tiene como objetivo \\ demostrar el poder explicativo de los medios nuevos y tradicionales en el proceso de la opinión \\ pública, con un fuerte enfoque en el análisis de datos de encuestas, el análisis de contenido y la \\ metodología de grupos focales. Su índice $\mathrm{H}$ es de 8 (enero de 2020). \\ arcastromil@,ccinf.ucm.es \\ Índice H: 8 \\ Orcid ID: https://orcid.org/0000-0001-9461-6647 \\ Google Scholar: https://scholar.google.es/citations?user=W2TpPFAAAAAJ\&hl=es
}

